\title{
Using phase-change materials to switch the direction of reflectionless light propagation in non-PT- symmetric structures
}

Georgios Veronis, Yin Huang, Yuecheng Shen, Changjun Min

Georgios Veronis, Yin Huang, Yuecheng Shen, Changjun Min, "Using phase-change materials to switch the direction of reflectionless light propagation in non-PT-symmetric structures," Proc. SPIE 10721, Active Photonic Platforms X, 107210I (19 September 2018); doi: 10.1117/12.2322004

EPIE Event: SPIE Nanoscience + Engineering, 2018, San Diego, California, United States 


\title{
Using phase-change materials to switch the direction of reflectionless light propagation in non-PT-symmetric structures
}

\author{
Georgios Veronis $^{\mathrm{a}, \mathrm{b}}$, Yin Huang ${ }^{\mathrm{c}}$, Yuecheng Shen ${ }^{\mathrm{d}}$, and Changjun Min ${ }^{\mathrm{e}}$ \\ ${ }^{a}$ School of Electrical Engineering and Computer Science, Louisiana State University, Baton Rouge, \\ Louisiana 70803, USA; \\ ${ }^{\mathrm{b}}$ Center for Computation and Technology, Louisiana State University, Baton Rouge, Louisiana \\ 70803, USA; \\ ${ }^{\mathrm{c}}$ Department of Optoelectrics Information Science and Engineering, School of Physics and \\ Electronics, Central South University, Changsha, Hunan 410012, China; \\ ${ }^{\mathrm{d} D e p a r t m e n t}$ of Medical Engineering, California Institute of Technology, Pasadena, California \\ 91125, USA; \\ ${ }^{\mathrm{e}}$ Key Laboratory of Optoelectronic Devices and Systems of Ministry of Education and Guangdong \\ Province, Shenzhen University, Shenzhen 518060, China
}

\begin{abstract}
We introduce a non-parity-time-symmetric three-layer structure, consisting of a gain medium layer sandwiched between two phase-change medium layers for switching of the direction of reflectionless light propagation. We show that for this structure unidirectional reflectionlessness in the forward direction can be switched to unidirectional reflectionlessness in the backward direction at the optical communication wavelength by switching the phase-change material $\mathrm{Ge}_{2} \mathrm{Sb}_{2} \mathrm{Te}_{5}$ (GST) from its amorphous to its crystalline phase. We also show that it is the existence of exceptional points for this structure with GST in both its amorphous and crystalline phases which leads to unidirectional reflectionless propagation in the forward direction for GST in its amorphous phase, and in the backward direction for GST in its crystalline phase. Our results could be potentially important for developing a new generation of compact active free-space optical devices. We also show that phase-change materials can be used to switch photonic nanostructures between cloaking and superscattering regimes at mid-infrared wavelengths. More specifically, we investigate the scattering properties of subwavelength three-layer cylindrical structures in which the material in the outer shell is the phase-change material GST. We first show that, when GST is switched between its amorphous and crystalline phases, properly designed electrically small structures can switch between resonant scattering and cloaking invisibility regimes. The contrast ratio between the scattering cross sections of the cloaking invisibility and resonant scattering regimes reaches almost unity. We then also show that larger, moderately small cylindrical structures can be designed to switch between superscattering and cloaking invisibility regimes, when GST is switched between its crystalline and amorphous phases. The contrast ratio between the scattering cross sections of cloaking invisibility and superscattering regimes can be as high as $\sim 93 \%$. Our results could be potentially important for developing a new generation of compact reconfigurable optical devices.
\end{abstract}

Keywords: Physical optics; Nanophotonics and photonic crystals; Guided waves; Physical optics; Scattering, invisibility; Subwavelength structures

\section{INTRODUCTION}

Exceptional points, which are branch point singularities of the spectrum, are associated with the coalescence of both eigenvalues and corresponding eigenstates in open quantum systems described by non-Hermitian Hamiltonians [1-7]. Exceptional points have been studied in lasers [8], coupled dissipative dynamical systems [9], mechanics [10], electronic circuits [11], and atomic as well as molecular systems [12]. In the past few years, unidirectional light reflectionlessness caused by the existence of exceptional points in non-Hermitian parity-time (PT) symmetric optical systems possessing balanced gain and loss has attracted considerable attention [13-17]. In such structures the reflection is zero when measured from one end of the structure at optical exceptional points, and nonzero when measured from the other end. Unidirectional light reflectionlessness can also be attained in non-PT-symmetric structures with unbalanced gain and

Active Photonic Platforms X, edited by Ganapathi S. Subramania, Stavroula Foteinopoulou,

Proc. of SPIE Vol. 10721, 107210I · (C) 2018 SPIE · CCC code: 0277-786X/18/\$18

doi: $10.1117 / 12.2322004$

Proc. of SPIE Vol. 10721 107210I-1 
loss [18-30]. This is due to the fact that exceptional points exist in a larger family of non-Hermitian Hamiltonians [18]. Achieving unidirectional reflectionless propagation is important for several key applications in photonic circuits such as optical network analyzers $[15,19]$. In addition, switching of the direction of reflectionless light propagation could be essential for building compact optoelectronic devices, for reducing the size of optical systems, and for developing reconfigurable optical components [31-33]. This could be achieved by using materials with tunable optical properties such as phase-change materials.



Fig. 1. Schematic of a non-PT-symmetric three-layer structure composed of a gain medium layer sandwiched between two GST layers for switching of the direction of reflectionless light propagation at exceptional points.

$\mathrm{Ge}_{2} \mathrm{Sb}_{2} \mathrm{Te}_{5}$ (GST) is a phase-change material with amorphous and crystalline phases [34]. The atom distribution of GST is chaotic in the amorphous phase. In contrast, the atoms are aligned in an orderly manner in the crystalline phase. Thus, GST can significantly change its optical properties through phase transitions. GST can be switched reversibly and rapidly between its amorphous and crystalline phases by applying external electrical pulses, optical pulses or thermal annealing. Picosecond-order crystallization times have been reported for GST by femtosecond laser pulses [35, 36]. Amorphization of GeSbTe has been achieved on the subpicosecond timescale with femtosecond laser pulse excitation [37]. In addition, GST retains its phase for years after removal of the external excitations. GST has been widely studied for applications in non-volatile, rewritable optical data storage and memory [38, 39]. Recently, a variety of optically reconfigurable GST-based active photonic devices have also been demonstrated [40-45].

Here, motivated by the transport behavior enabled by non-Hermiticity and the high refractive index contrast between the amorphous and crystalline phases of phase-change material GST, we use a non-PT-symmetric three-layer structure, consisting of a gain medium layer sandwiched between two GST layers, to switch the direction of reflectionless light propagation at exceptional points (Fig. 1). We show that, when GST is switched from its amorphous to its crystalline phase, the structure switches from unidirectional reflectionless in the forward direction to unidirectional reflectionless in the backward direction. The structure is designed at the optical communication wavelength of $\lambda 0=1.55 \mu \mathrm{m}$. We then discuss the underlying physical mechanism of unidirectional reflectionless light propagation in this structure. We show that a layer with gain has to be included in the structure to compensate the loss in the GST layers so as to achieve complete destructive interference. We demonstrate that the structure exhibits exceptional points for GST in both its amorphous and crystalline phases. These exceptional points result in unidirectional reflectionless propagation in the forward direction for GST in its amorphous phase, and in the backward direction for GST in its crystalline phase. We investigate the phase transitions associated with the exceptional points. Finally, the topological structure of the exceptional points is also explored by encircling them in parameter space.

In recent years, investigating the interaction of light with subwavelength structures has attracted a lot of attention, since it could potentially lead to a new generation of photonic devices [23, 46-50]. In particular, the capability to control the scattering of light and achieve invisibility cloaking of subwavelength structures is important for applications in biomedicine, photovoltaics, sensing, optical detection, and near-field imaging [51-57]. In the past few years, the use of plasmonic and dielectric multilayer coatings to drastically reduce the total scattering cross-section of deep subwavelength objects, and thus achieve invisibility cloaking based on scattering cancellation, has been explored [58-62]. In addition to cloaking, it has been demonstrated that subwavelength multilayer core-shell structures can lead to enhanced resonant scattering, so that the scattering cross sections of the original structures are greatly enhanced [63-66]. This phenomenon is commonly referred to as superscattering. Switching between the cloaking and enhanced scattering states could be essential for building compact optoelectronic devices, for reducing the size of optical systems, and for developing reconfigurable optical components [67-79]. Such switching between the enhanced scattering and invisibility cloaking regimes has been demonstrated using nonlinear materials [70] and quantum emitters [71]. An alternative way to achieve this switching could be through the use of materials with tunable optical properties, such as phase-change materials. 
Here, we also investigate the scattering properties of a three-layer cylindrical structure with GST at the mid-infrared wavelength of $4 \mu \mathrm{m}$ (Fig. 2). We first consider an electrically small structure. We show that, when GST is switched between its amorphous and crystalline phases, the structure switches between resonant scattering and cloaking invisibility regimes. The contrast ratio between the scattering cross sections of the cloaking invisibility and resonant scattering regimes reaches almost unity. We then consider the case of a larger, moderately small cylindrical structure. In this scenario, we demonstrate that, when GST is switched between its crystalline and amorphous phases, the structure switches between superscattering and cloaking invisibility regimes. The contrast ratio between the scattering cross sections of cloaking invisibility and superscattering regimes can be as high as $\sim 93 \%$. Although here we focus on two-dimensional infinitely long cylindrical structures, the proposed approach is rather general and can be applied to other optical structures.

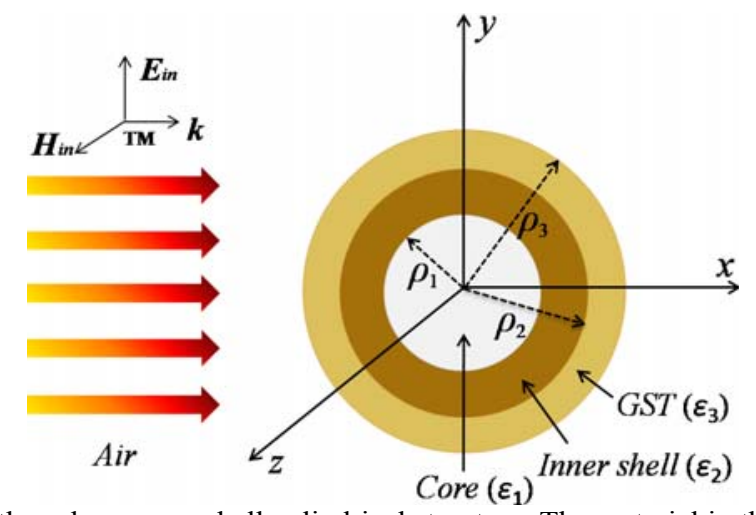

Fig. 2. Schematic of a three-layer core-shell cylindrical structure. The material in the outer shell is the phase-change material GST.

\section{CONCLUSIONS}

In this paper, we design a non-PT-symmetric three-layer structure, consisting of a gain medium layer sandwiched between two phase-change medium layers for switching of the direction of reflectionless light propagation at exceptional points. We show that for this structure unidirectional reflectionlessness in the forward direction can be switched to unidirectional reflectionlessness in the backward direction at the optical communication wavelength of $\lambda 0=$ $1.55 \mu \mathrm{m}$ by switching the phase-change material GST from its amorphous to its crystalline phase. We use the transfer matrix method and optimize the geometric and material parameters of the structure, to minimize the reflection in the forward direction when GST is in its amorphous phase, and the reflection in the backward direction when GST is in its crystalline phase. We also confirm these findings with full-wave finite-difference frequency-domain (FDFD) simulations. We discuss the underlying physical mechanism of unidirectional reflectionless light propagation in this structure, and the role of the ultrathin GST layer in the reflection process from the structure. We show that a gain material layer has to be included in the structure in order to compensate the material loss in the GST layers and achieve complete destructive interference.

In addition, for experimental realization, an ultrathin thermal barrier layer can be used next to a GST layer to confine heat in the GST film. Such a thermal barrier layer keeps surrounding materials isolated from heating and protects them from harmful interaction with the GST film, when GST is switched between its two phases by optically, electrically or thermally provided heat stimuli $[35,40,45]$. For example, $\mathrm{Ta}_{2} \mathrm{O}_{5}, \mathrm{Al}_{2} \mathrm{O}_{3}$ and $\mathrm{Si}_{3} \mathrm{~N}_{4}$ are materials which can be chosen for the thermal barrier due to their low thermal conductivities $[35,40,45]$. We find that the addition of such a thin thermal barrier layer does not affect the functionality of the proposed structure. In addition, even though the structure is designed assuming normally incident light, we find that the contrast ratio between the forward and backward reflection is large in a wide angular range for both TE and TM polarization, and for GST in both its amorphous and crystalline phases. Our results could be potentially important for developing a new generation of compact active free-space optical devices. It is noteworthy that the concept of combining gain and phase-change materials for switching of the direction of reflectionless light propagation could also be applied to nanoplasmonic waveguide-cavity systems [23, 26], which could lead to implementations in integrated optical chips. 
In this paper, we also design subwavelength three-layer core-shell cylindrical structures with the phase-change material GST for switching between the cloaking invisibility and enhanced scattering regimes at the mid-infrared wavelength of $\lambda 0=4 \mu \mathrm{m}$. We use the Mie-Lorenz mode-expansion method and optimize the geometric and material parameters of the structures to achieve the switching. For an electrically small three-layer structure, we optimize the dielectric permittivity of the material in the inner shell and the layer dimensions, to switch between cloaking and resonant scattering by switching the phase-change material GST from its crystalline to its amorphous phase. We find that for the optimized structure the contrast ratio between the cloaking and resonant scattering states is almost unity. For larger, moderately small three-layer structures, we optimize the layer dimensions to minimize the scattering cross section when GST is in its amorphous phase, and maximize the scattering cross section when GST is in its crystalline phase. We find that cloaking occurs when the amplitude of the monopole scattering coefficient is zero, while superscattering occurs when a higher order multipolar scattering mode is on resonance. For the optimized structure, the contrast ratio between the cloaking and superscattering states can be as high as $\sim 93 \%$. We also confirm these findings with full-wave finite-element simulations.

As final remarks, we first investigated a two-layer cylindrical structure consisting of a core layer and a GST shell layer. We found that such a two-layer structure cannot achieve the same functionality as the three-layer structure. In other words, the inner shell between the core and the GST outer shell is necessary to achieve switching between cloaking and enhanced scattering regimes. This is due to the fact that for electrically small two-layer cylindrical structures, a shell with negative or near-zero permittivity is necessary to cloak a dielectric core based on scattering cancellation [58, 59]. Thus, an electrically small two-layer structure consisting of a GST shell and a dielectric core cannot be used to realize switching between the cloaking and resonant scattering regimes. We also note that layered core-shell cylindrical nanostructures can be fabricated by chemical vapor deposition and sputter coating [72]. Light scattered from an individual cylindrical nanostructure can be detected using dark-field microscopy [73]. In addition, switching between cloaking and superscattering regimes using phase-change materials could also be generalized to three-dimensional spherical nanostructures [64]. Our results could be potentially important for developing a new generation of dynamically reconfigurable subwavelength optical devices.

Georgios Veronis acknowledges support from the National Science Foundation under award no. 1254934.

\section{REFERENCES}

[1] Moiseyev, M., Non-Hermitian Quantum Mechanics (Cambridge University, 2011).

[2] Heiss, W. D., "Exceptional points of non-Hermitian operators," J. Phys. Math. Gen. 37, 2455-2464 (2004).

[3] Heiss, W. D., "Repulsion of resonance states and exceptional points," Phys. Rev. E 61, 929-932 (2000).

[4] Lupu, A., Benisty, H. and Degiron, A., "Switching using PT-symmetry in plasmonic systems: positive role of the losses," Opt. Express 21, 21651-21668 (2013).

[5] Lupu, A., Benisty, H. and Degiron, A., "Using optical PT-symmetry for switching applications," Photon. Nanostructures 12, 305-311 (2014).

[6] Achilleos, V., Theocharis, G., Richoux, O. and Pagneux, V., "Non-Hermitian acoustic metamaterials: Role of exceptional points in sound absorption," Phys. Rev. B 95, 144303 (2017).

[7] Yu, S., Park, H., Piao, X., Min, B. and Park, N., "Low-dimensional optical chirality in complex potentials," Optica 3, 1025-1032 (2016).

[8] Berry, M. V., "Physics of nonhermitian degeneracies," Czech. J. Phys. 54, 1039-1047 (2004).

[9] Ryu, J. W., Son, W. S., Hwang, D. U., Lee, S. Y. and Kim, S. W., "Exceptional points in coupled dissipative dynamical systems," Nano Lett. 91, 052910 (2015).

[10] Kirillov, O. N., "Exceptional and diabolical points in stability questions," Fortschr. Phys. 61, 205-224 (2013).

[11] Stehmann, T., Heiss, W. S. and Scholtz, F. G., "Observation of exceptional points in electronic circuits," J. Phys. Math. Gen. 37, 7813-7819 (2004).

[12] Heiss, W. D., “The physics of exceptional points," J. Phys. A 45, 444016 (2012).

[13] Bender, C. M. and Boettcher, S., "Real spectra in non-Hermitian Hamiltonians having PT-symmetry," Phys. Rev. Lett. 80, 5243 (1998).

[14] Lin, Z., Ramezani, H., Eichelkraut, T., Kottos, T., Cao, H. and Christodoulides, D. N., "Unidirectional invisibility induced by PT-symmetric periodic structures," Phys. Rev. Lett. 106, 213901 (2011). 
[15] Regensburger, A., Bersch, C., Miri, M. A., Onishchukov, G., Christodoulides, D. N. and Peschel, U., "Parity-time synthetic photonic lattices," Nature 488, 167-171 (2012).

[16] Ge, L., Chong, Y. D. and Stone, A. D., "Conservation relations and anisotropic transmission resonances in one-dimensional PT-symmetric photonic heterostructures," Phys. Rev. A 85, 023802 (2012).

[17] Huang, Y., Shen, Y., Min, C., Fan, S. and Veronis, G., "Unidirectional reflectionless light propagation at exceptional points," Nanophotonics 6, 977-996 (2017).

[18] Yin, X. and Zhang, X., "Unidirectional light propagation at exceptional points," Nat. Mater. 12, 175-177 (2013).

[19] Feng, L., Xu, Y. L., Fegadolli, W. S., Lu, M. H., Oliveira, J. E. B., Almeida, V. R., Chen, Y. F. and Scherer, A., "Experimental demonstration of a unidirectional reflectionless parity-time metamaterial at optical frequencies," Nat. Mater. 12, 108-113 (2013).

[20] Feng, L., Zhu, X., Yang, S., Zhu, H., Zhang, P., Yin, X., Wang, Y. and Zhang, X., "Demonstration of a large-scale optical exceptional point structure," Opt. Express 22, 1760-1767 (2014).

[21] Shen, Y., Deng, X. H. and Chen, L., "Unidirectional invisibility in a two-layer non-PT-symmetric slab," Opt. Express 22, 19440-19447 (2014).

[22] Peng, B., Ozdemir, S. K., Liertzer, M., Chen, W., Johannes, K., Yilmaz, H., Wiersig, J., S, Rotter. and Yang, L., "Chiral modes and directional lasing at exceptional points," Proc. Natl. Acad. Sci. USA 113, 6845-6850 (2016).

[23] Huang, Y., Veronis, G., and Min, C., "Unidirectional reflectionless propagation in plasmonic waveguide-cavity systems at exceptional points," Opt. Express 23, 29882-29895 (2015).

[24] Jia, Y., Yan, Y., Kesava, S. V., Gomez, Z. D. and Giebink, N. C., "Passive parity-time symmetry in organic thin film waveguides," ACS Photonics 2, 319-325 (2015).

[25] Horsley, S. A. R., Artoni, M. and La Rocca, G. C., "Spatial Kramer-Kronig relations and the reflection of waves," Nat. Photonics 9, 436-439 (2015).

[26] Huang, Y., Min, C. and Veronis, G., "Broadband near total light absorption in non-PT-symmetric waveguide-cavity systems," Opt. Express 24, 22219-22231 (2016).

[27] Yang, E., Lu, Y., Wang, Y., Dai, Y. and Wang, P., "Unidirectional reflectionless phenomenon in periodic ternary layered material," Opt. Express 24, 14311-14321 (2016).

[28] Yu, S., Piao, X. and Park, N., "Acceleration toward polarization singularity inspired by relativistic E_B drift," Sci. Rep. 6, 37754 (2016).

[29] Yu, S., Piao, X., Yoo, K., Shin, J. and Park, N., "One-way optical modal transition based on causality in momentum space," Opt. Express 23, 24997-25008 (2015).

[30] Longhi, S., "Bidirectional invisibility in Kramers-Kronig optical media," Opt. Lett. 41, 2727-2730 (2016).

[31] Li, X., Tan, Q., Bai, B. and Jin, G., "Experimental demonstration of tunable directional excitation of surface Plasmon polaritons with a subwavelength metallic double slit," Appl. Phys. Lett. 98, 251109 (2011).

[32] Lin, J., Mueller, J. B., Wang, Q., Yuan, G., Antoniou, N., Yuan, X. and Capasso, F., "Polarization-controlled tunable directional coupling of surface plasmon polaritons," Science 340, 331-334 (2013).

[33] Kim, S., Yun, H., Park, K., Hong, J., Yun, J., Lee, K., Kim, J., Jeong, S., Mun, S., Sung, J., Lee, Y. and Lee, B., "Active directional switching of surface plasmon polaritons using a phase transition material," Sci. Rep. 7, 43723 (2017).

[34] Shportko, K., Kremers, S., Woda, M., Lencer, D., Robertson, J. and Wuttig, M., "Resonant bonding in crystalline phase-change materials," Nat. Mater. 7, 653-658 (2008).

[35] Rude, M., Mkhitaryan, V., Cetin, A. E., Miller, T. A., Carrilero, A., Wall, S., Abajo, F. J., Altug, H. and Pruneri, V., "Ultrafast and broadband tuning of resonant optical nanostructures using phase-change materials," Adv. Optical Mater.

4, 1060-1066 (2016).

[36] Loke, D., Lee, T. H., Wang, W. J., Shi, L. P., Zhao, R., Yeo, Y. C., Chong, C. T. and Elliott, S. R., "Breaking the speed limits of phase-change memory," Science 336, 1556-1569 (2012).

[37] Hira, T., Homma, T., Uchiyama, T., Kuwamura, K., Kihara, Y. and Saiki, T., "All-optical switching of localized surface plasmon resonance in single gold nanosandwich using GeSbTe film as an active medium,” Appl. Phys. Lett. 106, 031105 (2015).

[38] Wuttig M. and Yamada, N., "Phase-change materials for rewriteable data storage," Nat. Mater. 6, 824-832 (2007).

[39] Lankhorst, M. H. R., Ketelaarsand, B. W. and Wolters, R. A. M., "Low-cost and nanoscale non-volatile memory concept for future silicon chips," Nat. Mater. 4, 347-352 (2005).

[40] Schlich, F. F., Zalden, P., Lindenberg, A. M. and Spolenak, R. "Color switching with enhanced optical contrast in ultrathin phase change materials and semiconductors induced by femtosecond laser pulses," ACS Photonics 2, 178-182 (2015).

[41] Rude, M., Simpson, R. E., Quidant, R., Pruneri, V. and Renger, J., "Active control of surface plasmon waveguides with a phase change material," ACS Photonics 2, 669-674 (2015). 
[42] Wang, Q., Rogers, E. T. F., Gholipour, B., Wang, C., Yuan, G., Teng and J., Zheludev, N. I., "Optically reconfigurable metasurfaces and photonic devices based on phase change materials," Nat. Photonics 10, 60-65 (2016). [43] Li, P., Yang, X., Mab, T. W. W., Hanss, J., Lewin, M., Michel, A. U., Wuttig, M. and Taubner, T., "Reversible optical switching of highly confined phonon-polaritons with an ultrathin phase-change material," Nat. Mater. 15, 870-876 (2016).

[44] Mkhitaryan, V. K., Ghosh, D. S., Rude, M., Canet-Ferrer, J., Maniyara, R. A., Gopalan, K. K. and Pruneri, V., "Tunable complete optical absorption in multilayer structures including Ge2Sb2Te5 without lithographic patterns," Adv. Optical Mater. 5, 1600452 (2017).

[45] Yoo, S., Gwon, T., Eom, T., Kim, S. and Hwang, C., "Multicolor changeable optical coating by adopting multiple layers of ultrathin phase change material film," ACS Photonics 3, 1265-1270 (2016).

[46] Novotny, L. and Hecht, B., Principles of Nano-Optics (Cambridge University, 2006).

[47] Barnes, W. L., Dereux, A. and Ebbesen, T. W., "Surface plasmon subwavelength optics," Nature 424, 824-830 (2003).

[48] Lal, S., Link, S. and Halas, N. J., "Nano-optics from sensing to waveguiding," Nat. Photonics 1, 641-648 (2007).

[49] Stockman, M. I., "Nanoplasmonics: past, present, and glimpse into future," Opt. Express 19, 22029-22106 (2011).

[50] Yang, Z., Jiang, R., Zhuo, X., Xie, Y., Wang, J. and Lin, H., "Dielectric nanoresonators for light manipulation," Phys. Rep. 701, 1-50 (2017).

[51] Hirsch, L. R., Stafford, R. J., Bankson, J. A., Sershen, S. R., Rivera, B., Price, R. E., Hazle, J. D., Halas, N. J. and West, J. L., "Nanoshell-mediated near-infrared thermal therapy of tumors under magnetic resonance guidance," Proc. Natl. Acad. Sci. USA 100, 13549-13543 (2003).

[52] Jackson, J. B. and Halas, N. J., "Surface-enhanced Raman scattering on tunable plasmonic nanoparticle substrates," Proc. Natl. Acad. Sci. USA 101, 17930-17935 (2004).

[53] Atwater, H. A. and Polman, A., "Plasmonics for improved photovoltaic devices," Nat. Mater. 9, 205-213 (2010).

[54] Alu, A. and Engheta, N., "Cloaking a sensor," Phys. Rev. Lett. 102, 233901 (2009).

[55] Fan, P., Chettiar, U. K., Cao, L., Afshinmanesh, F., Engheta, N. and Brongersma, M. L., "An invisible metal-semiconductor photodetector," Nat. Photonics 6, 380-385 (2012).

[56] Alu, A. and Engheta, N., "Cloaked near-field scanning optical microscope tip for noninvasive near-field imaging," Phys. Rev. Lett. 105, 263906 (2010).

[57] Mirzaei, A., Miroshnichenko, A. E., Shadrivov, I. V., and Kivshar, Y. S., “Optical metacages,” Phys. Rev. Lett. 115, $215501(2015)$.

[58] Alu, A. and Engheta, N., "Achieving transparency with plasmonic and metamaterial coatings," Phys. Rev. E 72, 016623 (2005).

[59] Chen, P., Soric, J. and Alu, A., "Invisibility and cloaking based on scattering cancellation," Adv. Mater. 24, OP281-OP304 (2012).

[60] Mirzaei, A., Miroshnichenko, A. E., Shadrivov, I. V. and Kivshar, Y. S., "All-dielectric multilayer cylindrical structures for invisibility cloaking," Sci. Rep. 5, 9574 (2015).

[61] Muhlig, S., Farhat, M., Rockstuhl, C. and Lederer, F., "Cloaking dielectric spherical objects by a shell of metallic nanoparticles," Phys. Rev. B 83, 195116 (2011).

[62] Monti, A., Bilotti, F. and Toscano, A., "Optical cloaking of cylindrical objects by using covers made of core-shell nanoparticles," Opt. Lett. 36, 4479-4481 (2011).

[63] Ruan, Z. and Fan, S., "Superscattering of light from subwavelength nanostructures," Phys. Rev. Lett. 105, 013901(2010).

[64] Ruan, Z. and Fan, S,. "Design of subwavelength superscattering nanospheres," Appl. Phys. Lett. 98, 043101(2011).

[65] Mirzaei, A., Miroshnichenko, A. E., Shadrivov, I. V. and Kivshar, Y. S., "Superscattering of light optimized by a genetic algorithm," Appl. Phys. Lett. 105, 011109 (2014).

[66] Huang, Y. and Gao, L., "Superscattering of light from core-shell nonlocal plasmonic nanoparticles," J. Phys. Chem. C 118, 30170-30178 (2014).

[67] Li, X., Tan, Q., Bai, B. and Jin, G., "Experimental demonstration of tunable directional excitation of surface Plasmon polaritons with a subwavelength metallic double slit," Appl. Phys. Lett. 98, 251109 (2011).

[68] Lin, J., Mueller, J. B., Wang, Q., Yuan, G., Antoniou, N., Yuan, X. and Capasso, F., "Polarization-controlled tunable directional coupling of surface plasmon polaritons," Science 340, 331-334 (2013).

[69] Kim, S., Yun, H., Park, K., Hong, J., Yun, J., Lee, K., Kim, J., Jeong, S., Mun, S., Sung, J., Lee, Y. and Lee, B., "Active directional switching of surface plasmon polaritons using a phase transition material," Sci. Rep. 7, 43723 (2017). 
[70] Argyropoulos, C., Chen, P., Monticone, F., Aguanno, G. D. and Alu, A., "Nonlinear plasmonic cloaks to realize giant all-optical scattering switching," Phys. Rev. Lett. 108, 263905 (2012).

[71] Chen, X., Sandoghdar, V. and Agio, M., "Coherent interaction of light with a metallic structure coupled to a single quantum emitter: from superabsorption to cloaking," Phys. Rev. Lett. 110, 153605 (2013).

[72] Zhang, L., Tu, R. and Dai, H., "Parallel core-shell metal-dielectric-semiconductor germanium nanowires for high-current surround-gate field-effect transistors," Nano Lett. 6, 2785-2789 (2006).

[73] Wiecha, P. R., Cuche, A., Arbouet, A., Girard, C., Francs, G., Lecestre, A., Larrieu, G., Fournel, F., Larrey, V., Baron, T. and Paillard, V., "Strongly directional scattering from dielectric nanowires," ACS Photonics 4, 2036-2046 (2017). 